\title{
Surface electronic and magnetic properties of semiconductor $\mathrm{FeSi}$ 文
}

\author{
G.Y. Guo * \\ Department of Physics, National Taiwan University, Taipei 10617, Taiwan, ROC
}

\begin{abstract}
First-principles local spin-density functional calculations of the electronic and magnetic properties of semiconducting FeSi (1 111 ) surface with both Fe- and Si-terminations have been performed. It is found that the Fe-terminated (1 111 ) surface is strongly ferromagnetic with a magnetic moment of $2.6 \mu_{\mathrm{B}} /$ atom on the top Fe monolayer while the Si-terminated (1 111$)$ surface is only weakly magnetic or nonmagnetic. These interesting results suggest that a semiconductor-based spintronics device might be developed by using thin FeSi (1 111$)$ films connected to a metal lead on the Fe-terminated surface and to a semiconductor substrate on the Si-terminated surface. (c) 2001 Elsevier Science B.V. All rights reserved.
\end{abstract}

PACS: 75.30.Pd; 75.50.Pp; 75.70.Cn

Keywords: Surface; Thin films; FeSi; Magnetism; Density of states

Bulk transition metal silicides have been intensively studied in the past decades due to their high-temperature stability, resistance to degradation, high electrical conductivity, and unusual magnetism [1-7]. In particular, the latter quality make one member of the silicide family, viz., FeSi, especially interesting for possible spintronics applications. The simple cubic compound FeSi has four $\mathrm{Fe}$ and four $\mathrm{Si}$ atoms per unit cell and its crystal structure is

\footnotetext{
Presented at PASPS 2000 (No. 109).

* Corresponding author. Tel.: +886-2-23626937; fax: +886-2-23639984.

E-mail address: gyguo@phys.ntu.edu.tw (G.Y. Guo).
}

$B 20$ (Fig. 1). It is a small gap semiconductor at low temperatures [4] and a highly enhanced paramagnet or a nearly ferromagnet at high temperatures $[2,3]$. Thus, it is conceivable that if a surface is created, the Fe atoms on the top layer and possibly the sublayers could become magnetic. Also, Fig. 1 shows that (1 111 ) atomic layers each consists of either purely $\mathrm{Fe}$ atoms or $\mathrm{Si}$ atoms. One thus could fabricate a thin FeSi film with one Fe-terminated surface and the other Si-terminated. One could then make semiconductor-based magneto-electronic components by connecting the Si-terminated surface to a Si substrate and the Fe-terminated surface to a metallic lead. Motivated by this interesting observation, I have carried out first-principles theoretical investigations 


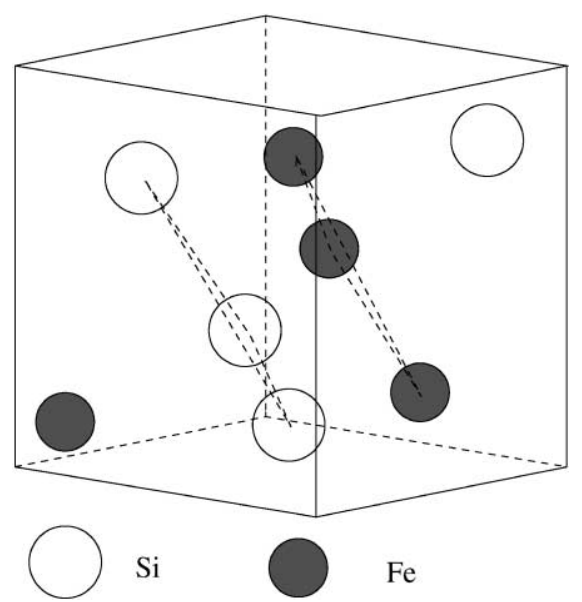

Fig. 1. Crystal structure of simple cubic FeSi.

of the electronic and magnetic properties of $\mathrm{FeSi}$ (1 111$)$. Indeed, I found that the Fe-terminated surface is ferromagnetic. This suggests that thin FeSi (1 111$)$ films could find their use in highly spin-polarization current source and other magneto-electronic devices.

I modelled FeSi ( $\left.\begin{array}{lll}1 & 1 & 1\end{array}\right)$ surfaces by a slab-supercell of three [ $\left.\begin{array}{lll}1 & 1 & 1\end{array}\right]$ cubic units along the surface normal ( $z$-axis) with a two-unit FeSi slab and a one-unit vacuum layer. The slab contains $24 \mathrm{Fe}$ monolayers and 24 Si monolayers with one Fe-terminated surface and the other Si-terminated surface. In this way, we can study both types of FeSi ( 1111$)$ surfaces in one calculation. A 48 monolayer thick slab is perhaps sufficiently thick to simulate the FeSi ( 1111 ) surfaces. No lattice relaxation is considered in this work. Thus the resultant superlattice has a trigonal symmetry with hexagonal primitive vectors. The lateral lattice constant $(a)$ is $\sqrt{2}$ times that of cubic FeSi and the longitudinal lattice constant $(c)$ is $3 \sqrt{\frac{3}{2}} a$. Lattice relaxation could occur and could affect the electronic and magnetic properties of $\mathrm{FeSi}$ ( $\left.1 \begin{array}{lll}1 & 1 & 1\end{array}\right)$ surfaces significantly. Investigations of the effects of lattice relaxation are under way and will be reported in a future publication. The self-consistant spin-polarized electronic structure has been calculated by using the fast linear muffin-tin orbital (LMTO) method with the atomic sphere approximation [8]. The atomic sphere radius used for the $\mathrm{Fe}$ atoms is that of bulk BCC Fe (2.662 a.u.) and that for the Si atoms is 2.6 a.u., determined by the experimental equilib-
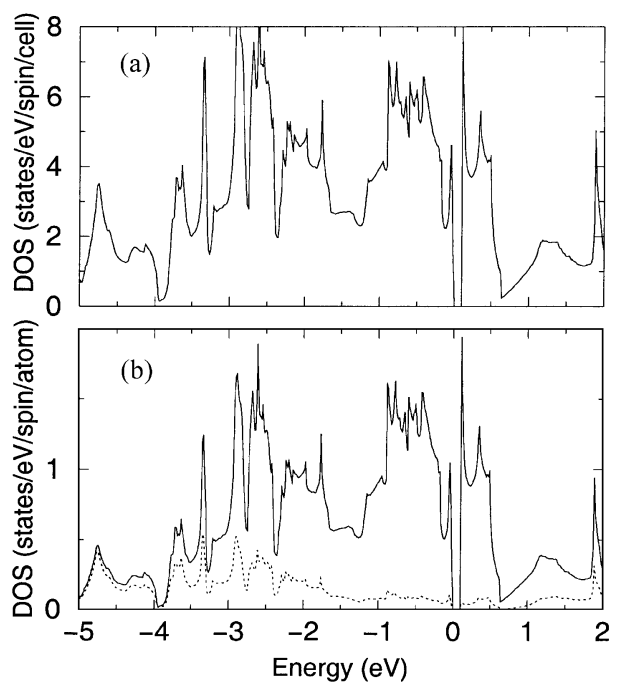

Fig. 2. Total (a) and Fe- and Si-site (b) local density of states (DOS) of bulk cubic FeSi. The Fermi level is at the zero energy.

rium volume of bulk FeSi minus the Fe atomic sphere volume. The vacuum layer is filled with 12 'empty' sphere layers of the $\mathrm{Fe}$ atomic size and 12 'empty' spheres of the Si atomic size. The wave function basis set consists of spd MTO orbitals for the Fe and Si atoms and sp MTO orbitals for the 'empty' spheres. The so-called combined correction terms are included [8]. The accurate local exchange-correlation potential of Vosk et al. [9] was used. The analytic tetrahedron method [10] is used for the Brillouin zone (BZ) integration with $72 k$-points over the irreducible wedge (IW) $\left(\frac{2}{12}\right.$ of BZ) for the self-consistent calculations and 576 for the final density of states calculations. For comparison, the electronic band structure of bulk FeSi has also been calculated. In the bulk self-consistent calculations and the final density of states calculation, 165 and $455 k$-points over the IWBZ $\left(\frac{1}{48}\right)$ were used, respectively.

Fig. 2 displays the calculated total and sitedecomposed density of states (DOS) of bulk cubic FeSi. The calculated total DOS spectrum is similar to that obtained by the highly accurate full-potential linear augmented plane wave (FLAPW) method [5] and almost identical to that obtained by a previous LMTO calculation [7]. Fig. 2 shows that bulk FeSi is a semiconductor with a small gap of $0.1 \mathrm{eV}$, in agreement with low-temperature experiments [4] and 


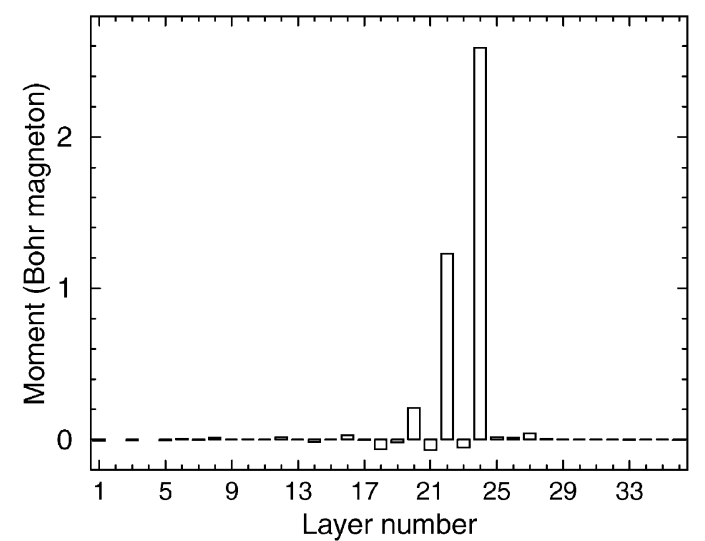

Fig. 3. Layer-decomposed magnetic moments of a (1 111$)$ supercell of FeSi. Layer no. $2 n(n=1-12)$ are $\mathrm{Fe}$ atomic layers, layer no. $2 n-1(n=1,12)$ are $\mathrm{Si}$ atomic layers and layer no. 25-36 are vaccuum layers.

previous calculations [5,7]. The density of states rises sharply to a high value on both sides of the gap, giving rise to highly enhanced magnetic susceptibility $[2,3,7]$.

Calculated layer-decomposed atomic magnetic moments are shown in Fig. 3. In the Fe-terminated surface, the top $\mathrm{Fe}$ monolayer is ferromagnetic with a considerable magnetic moment of $2.6 \mu_{\mathrm{B}} /$ atom. This magnetic moment is larger than that of bulk BCC $\mathrm{Fe}\left(2.2 \mu_{\mathrm{B}} /\right.$ atom $)$. The magnetic moments, however, decrease rapidly as one moves inwards, and in the first subsurface Fe monolayer, the magnetic moment is already reduced to about one third of that on the top Fe layer. The magnetic moment becomes neglible beyond the second subsurface Fe layer (Fig. 3). In the Si-terminated surface, the first Fe monolayer is only weakly ferromagnetic with a small moment of $0.2 \mu_{\mathrm{B}} /$ atom and the magnetic moment becomes completely neglible beyond the first subsurface Fe atomic layer. The Si-terminated surface could be nonmagnetic if a much thicker slab had been used. The presence of the small magnetic moments in the first $\mathrm{Fe}$ monolayer in the Si-terminated surface instead indicates that there is a weak exchange coupling between the Fe monolayers on the both sides of the thin film, despite of the fact that $\mathrm{FeSi}$ is a semiconductor and the two Fe monolayers are $14 \AA$ apart. The magnetic moments on the $\mathrm{Si}$ atoms are generally small (within $0.01 \mu_{\mathrm{B}} /$ atom) except the first and second subsurface layers in the Fe-terminated surface (Fig. 3 ) in which the magnetic moment values amount to $0.07 \mu_{\mathrm{B}} /$ atom. Interestingly, most magnetic moments on the $\mathrm{Si}$ atoms are antiparalell to that on the Fe atomic layers. The total magnetic moment per supercell is $10.1 \mu_{\mathrm{B}}$.

Calculated layer- and spin-decomposed densities of states (DOS) are plotted in Fig. 4 for both Fe-terminated (a) and Si-terminated (b) surfaces. Clearly, in the case of Fe-terminated surface, the DOS of the top Fe layer is strongly exchange-split (Fig. 4a). In fact, the DOS spectrum is similar to that of a $\mathrm{Fe}$ impurity in noble metals [11]. The exchange-splitting is about $2 \mathrm{eV}$ in both cases. Like the magnetic moments, these spin-splittings drop dramatically as one goes inwards (up-down in Fig. 4a) and in the second subsurface layer, they become nearly undiscernable. The shape of the DOS in the top Fe layer is also significantly different from that of the second subsurface layer and inwards. The DOS spectrum of the third sublayer and below is similar to that of the innermost (sixth) layer. The DOS spectra of the innermost layers (Fig. 4) resemble that of bulk FeSi (Fig. 2 ), though they are a bit noisy perhaps due to the insufficient number of $k$-points used. Thus the DOS of the fourth and fifth subsurface layers are not shown in Fig. 4. An interesting finding is that the layer- and spin-decomposed DOS spectra near the Fermi level are highly polarized (Fig. 4) due to the presence of the ferromagnetic surface. In other words, in this thin film system, the electrons around the Fermi level which dominate electronic transport processes, would be highly spin-polarized surface layers (Fig. 4). This therefore would suggest that thin FeSi (1 111$)$ films could be used as a spin-filter and other magneto-electronic devices. Of course, further experimental and/or theoretical work is needed in order to realize this proposition. In the case of Si-terminated surface, the exchange-splitting is small though the DOS spectrum of the first $\mathrm{Fe}$ monolayer is also significantly different from that of the subsurface and inner $\mathrm{Fe}$ monolayers (Fig. 4b).

In conclusion, motivated by the proposed application of thin FeSi (1 111 ) films in semiconductor-based spintronics due to its alternating Fe and Si layer structure along [1 111 ], I have performed first-principles local spin-density functional calculations of the elec- 

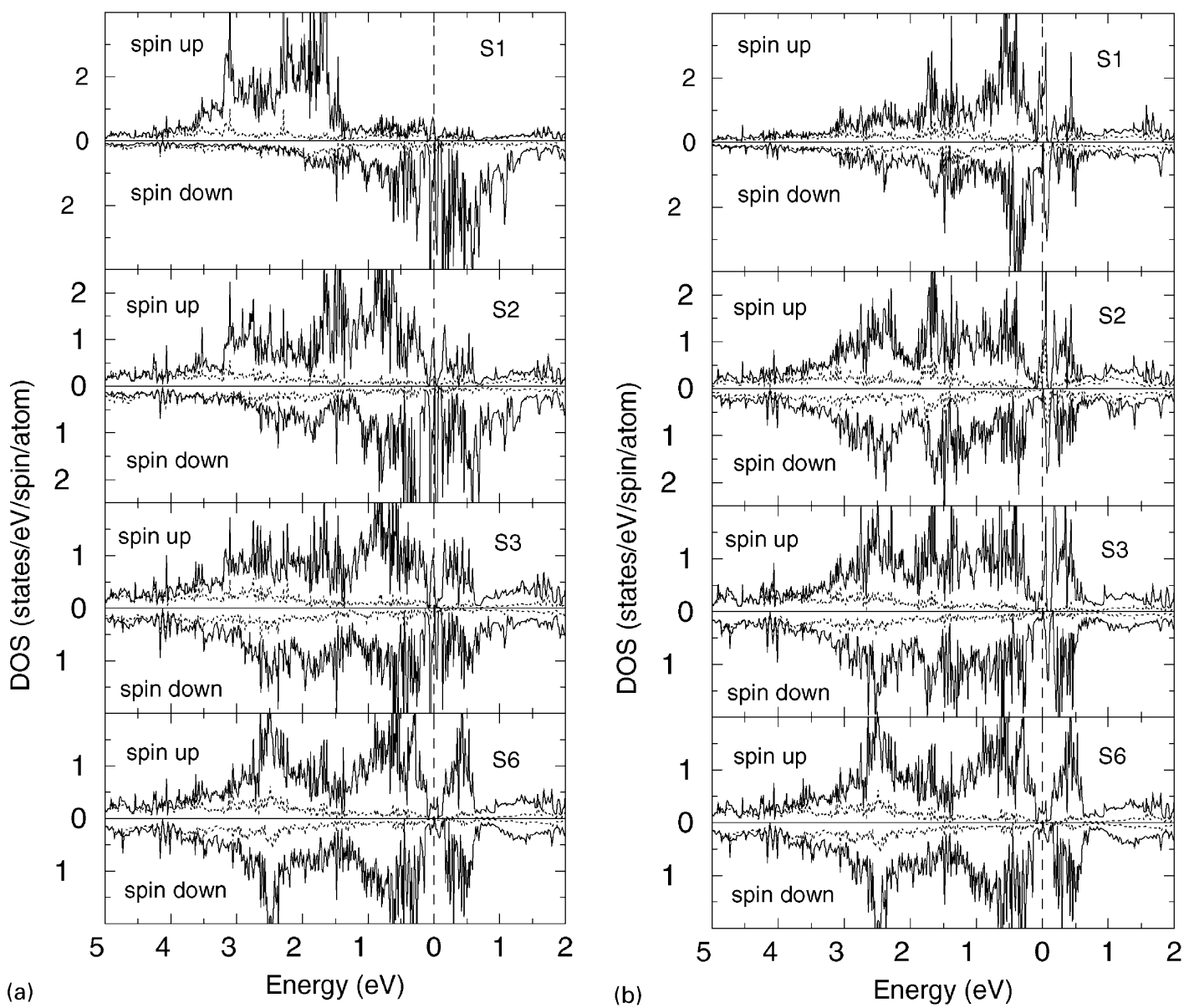

Fig. 4. Layer-decomposed density of states (DOS) of a (1111) supercell of FeSi. The solid lines represent that of Fe atomic layers and the dotted lines the Si atomic layers. S $n$ denotes the $n$th atomic layers from the surface. The Fe-terminated surface is displayed in (a) and the Si-terminated in (b). The Fermi level is at the zero energy.

tronic and magnetic properties of semiconducting FeSi (1 111 ) surface with both Fe- and Si-terminations. It is found that the Fe-terminated (1 111 ) surface is ferromagnetic with a large magnetic moment of $2.6 \mu_{\mathrm{B}} /$ atom on the top $\mathrm{Fe}$ monolayer while the Si-terminated (1 111 ) surface is only weakly magnetic or nonmagnetic. These interesting results strongly indicate that it would be possible to develop semiconductor-based magneto-electronic devices using thin FeSi (1 111$)$ films.

The author acknowledges financial support from the National Science Council of Republic of China (NSC 89-2112-M-002-0025).

\section{References}

[1] K.N. Tu, J.W. Mayer, in: J.M. Poate, K.N. Tu, J.W. Mayer (Eds.), Thin Films Interdiffusion and Interation, Wiley, New York, 1978.

[2] V. Jaccarino et al., Phys. Rev. 160 (1976) 476.

[3] G. Shirane et al., Phys. Rev. Lett. 59 (1987) 351.

[4] Z. Schlesinger et al., Phys. Rev. Lett. 71 (1993) 1748.

[5] L.F. Mattheiss, D.R. Hamann, Phys. Rev. B 47 (1993) 13114.

[6] S. Eisebitt et al., Phys. Rev. B 50 (1994) 18330.

[7] T. Jarlborg, Phys. Rev. B 51 (1995) 11106.

[8] O.K. Andersen, Phys. Rev. B 12 (1975) 3060.

[9] S.H. Vosko, L. Wilk, M. Nusair, Can. J. Phys. 58 (1980) 1200.

[10] W.M. Temmerman, P.A. Sterne, G.Y. Guo, Z. Szotek, Mol. Simulation 4 (1989) 153.

[11] G.Y. Guo, Phys. Rev. B 62 (2000) R14609. 